\title{
Resveratrol Treatment Is Associated with Lipid Regulation and Inhibition of Lipoprotein-Associated Phospholipase A2 (Lp-PLA2) in Rabbits Fed a High-Fat Diet
}

\author{
Lei Xu $\mathbb{D}^{D},{ }^{1}$ Renjie Wang, ${ }^{2}$ Hongyu Liu, ${ }^{1}$ Jiaoqi Wang, ${ }^{1}$ Jing Mang $\mathbb{C}^{1},{ }^{1}$ and Zhongxin Xu $\mathbb{C D}^{1}$ \\ ${ }^{1}$ Department of Neurology, China-Japan Union Hospital of Jilin University, Changchun City, Jilin Province 130033, China \\ ${ }^{2}$ Department of Nuclear Medicine, China-Japan Union Hospital of Jilin University, Changchun City, \\ Jilin Province 130033, China
}

Correspondence should be addressed to Jing Mang; mangjing@jlu.edu.cn and Zhongxin Xu; xuzhongxin186@163.com

Received 29 November 2019; Revised 10 March 2020; Accepted 18 April 2020; Published 20 May 2020

Academic Editor: Armando Zarrelli

Copyright (C) 2020 Lei Xu et al. This is an open access article distributed under the Creative Commons Attribution License, which permits unrestricted use, distribution, and reproduction in any medium, provided the original work is properly cited.

\begin{abstract}
The effects of resveratrol on various conditions have been widely studied previously. This paper aimed to investigate the influence of resveratrol on atherosclerosis (AS). Twenty-four New Zealand male rabbits were randomly and equally assigned to the normal diet group (NDG), fat diet group (FDG), and fat diet with resveratrol group ( $80 \mathrm{mg} / \mathrm{kg} / \mathrm{d}, \mathrm{RFG})$. Biochemical indicators from blood samples were analyzed at baseline and 3 months to investigate the effects of resveratrol on blood lipid, lipoprotein-associated phospholipase A2 (Lp-PLA2), liver, and renal function. The indicators including alanine aminotransferase (ALT), aspartate aminotransferase (AST), creatinine (CREA), triglycerides (TG), total cholesterol (TC), high-density lipoprotein cholesterol (HDL-C), low-density lipoprotein cholesterol (LDL-C), and Lp-PLA2. At 3 months, arteries were stained with hematoxylin and eosin to study the influence of resveratrol on the aortic intima, smooth muscle layer, and the intima/media ratio. Comparisons of weight, ALT, AST, CREA, TG, TC, HDL-C, LDL-C, and Lp-PLA2 among the three groups showed no significant difference at baseline. However, at the end of 3 months, significant differences were observed in AST, CREA, TC, HDL-C, LDL-C, and Lp-PLA2 between the three groups $(P<0.05)$. In pairwise comparison, CREA, TC, LDL-C, and Lp-PLA2 had significant differences between any two groups $(P<0.05)$. In addition, there were significant differences in the AST and HDL-C levels between RFG and NDG groups $(P<0.05)$. Meanwhile, the HDL-C levels were also significantly different between the FDG and NDG groups $(P<0.01)$. The histologic analysis also showed that the thickness of the aortic intima and the ratio of the intima and aortic tunica media $(P<0.05)$ significantly decreased in RFG compared to FDG. Resveratrol may have an antiatherosclerosis effect on a rabbit model of AS.
\end{abstract}

\section{Introduction}

Atherosclerosis (AS) is a disease that seriously endangers human health. Numerous basic and clinical studies indicate that AS is a chronical inflammatory disease, characterized by an abnormal reaction to the damage of blood vessel walls, with symptoms including classic inflammatory degeneration, exudation, and proliferation characteristics [1]. AS can induce a variety of fatal ischemic cardio-cerebrovascular diseases, such as cerebrovascular disease, coronary heart disease, and thromboembolic disease [2]. With the increase in life expectancy caused by improved living standards, more attention has been put on the damage caused by cardiovascular and cerebrovascular diseases [3].

Resveratrol is a polyphenolic compound that can be naturally produced by numerous plants, including grapes, peanuts, and mulberries [4]. There are different opinions about the effect of resveratrol on atherosclerosis. A study by Wilson showed that resveratrol promotes atherosclerotic development, rather than protecting against it, by a mechanism that is independent of observed differences in gross animal health, liver function, plasma cholesterol concentrations, or low-density lipoprotein (LDL) oxidative status [5]. However, numerous studies both in vitro and in 
vivo have shown that resveratrol can protect against atherosclerotic cardiovascular disease through several potential mechanisms, including mitigation of inflammation [6] or oxidative stress [7], regulation of energy metabolism [8], enhancement of endothelial function and vasorelaxation [9], and inhibition of platelet aggregation [10]. In vitro cell culture studies have also shown that while inhibiting foam cell formation, resveratrol can also improve the metabolism of lipoproteins and reverse the transportation of cholesterol $[11,12]$.

However, it is still controversial whether resveratrol can improve AS and alter blood lipids and function of liver and kidney. There are also limited data concerning the effect of resveratrol on blood lipoprotein-associated phospholipase A2 (Lp-PLA2). Lp-PLA2, also known as platelet-activating factor acetylhydrolase (PAF-AH), is a $45 \mathrm{kD}$, calcium-independent enzyme which "belongs to the phospholipase A2 superfamily. Lp-PLA2 can be produced by inflammatory cells in atherosclerotic plaques, such as monocytes, macrophages, T-lymphocytes, and mast cells $[13,14]$. In blood circulation, under the effect of chemotactic inflammatory cells, Lp-PLA2 generates a self-reinforcing positive feedback cycle, generating proinflammatory substances and leading to the occurrence and development of atherosclerosis $[15,16]$. A study by Sun revealed that resveratrol caused a decrease in macrophage Lp-PLA2 levels and reduced the infiltration of inflammatory cells in the mouse liver [17]. A study by Luo showed that resveratrol assumed a protective position adjacent to the phospholipid head group of the phospholipid bilayers, proximal to fatty acyl chains, and was able to protect phospholipids from hydrolytic attack by PLAs [18]. However, the effect of resveratrol on Lp-PLA2 levels in an AS model remains to be investigated. Due to the important role of Lp-PLA2 in the development of AS, it is necessary to further study the regulation of resveratrol on Lp-PLA2 in AS. The activities of AS, an inflammatory disease, can be assessed by measuring the levels of circulating biomarkers. Sufficient evidence has been accumulated for Lp-PLA2 to be used as a biomarker in clinical practice $[19,20]$. With a high specificity for vascular inflammation and a direct role in the causal pathway of plaque inflammation, Lp-PLA2 is relatively unique in its high specificity, making it an exemplary biomarker [21-23].

Two main animal AS models have been developed for the study of the molecular mechanisms underlying AS: one is induced by a high-fat diet and another involves intimal injury $[10,24]$. Due to the experimental limitations of the intimal injury method, caused by restrictions of the equipment, the fat diet is considered to more accurately follow the chronic progression of human AS, although it requires more time. Thus, in the present study, we utilized the fat diet rabbit AS model to investigate the influence of resveratrol on blood lipids, Lp-PLA2, liver/kidney function, and AS.

\section{Materials and Methods}

2.1. Ethical Statement. All experiments were approved by the Institutional Animal Care and Use Committee at the
China-Japan Union Hospital of Jilin University. All procedures were performed in accordance with the National Institute of Health's Guide for the Care and Use of Laboratory Animals.

2.2. Rabbits. Three-month-old New Zealand male white rabbits, weighing approximately $2 \mathrm{~kg}$, were obtained from Changchun Yis Laboratory Animal Technology (Jilin, China). The rabbits were given free access to food and water, while housed under a natural day/night cycle.

To test the hypothesis that the fat diet can induce AS, a preliminary experiment was conducted. A control (NDG) rabbit was fed on a normal diet (NDG), while an experimental (FDG) rabbit fed was fed on a fat diet. The normal diet contained carbohydrates $68 \%$, protein $22 \%$, salt $0.5 \%$, and fat $10 \%$, and the fat diet contained $1 \%$ cholesterol, $5 \%$ lard, 5\% yolk powder, and $89 \%$ basic rabbit feed (Beijing Botai Hongda Biotechnology Co., Ltd., China). The rabbits were maintained on this diet for 3 months. The aortic arch was harvested from the rabbits after euthanasia by injection with sodium phenobarbital $(0.2 \mathrm{~g} / \mathrm{kg}$, diluted concentration $3.5 \%$ ) into the ear vein and put in a test tube containing $4 \%$ paraformaldehyde for histological evaluation. Perpendicular to the vascular axis, the aortic arch was cut into approximately $2 \mathrm{~mm}$ lengths, embedded in paraffin and stained with hematoxylin and eosin. Atherosclerotic lesions were detected by light microscopy in the rabbit fed on a fat diet. This model has previously been used for studies of AS $[25,26]$. As shown in Figure 1, foam cells were formed under the intima, the intima was thickened, and plaques were formed in the FDG group rabbit. This preliminary experiment offered evidence that the high-cholesterol diet was capable of causing atherosclerosis.

Twenty-four rabbits were randomly assigned to three groups: a normal diet group (NDG) served as a control; a fat diet group (FDG); and an FDG group treated with resveratrol (RFG). The treatment concentrations of resveratrol were determined according to a preliminary experiment and a literature review (Beijing Solarbio Science \& Technology Co., Ltd., China). Resveratrol ( $80 \mathrm{mg} / \mathrm{kg} /$ day) was mixed in with the feed ( $50 \mathrm{~g}$, twice a day) and accounted for about $0.2 \%$ of the feed $[17,27]$.

2.3. Blood Chemistry. Blood samples were collected by heart puncture from the rabbits in the three groups at the baseline, before commencement of the diet in the morning, and after 3 months. The determinations of blood levels of alanine aminotransferase (ALT, IU/L), aspartate aminotransferase (AST, IU/L), creatinine (CREA, $\mu \mathrm{mmol} / \mathrm{L})$, triglycerides (TG, mmol/L), total cholesterol (TC; mmol/L), high-density lipoprotein cholesterol (HDL-C, mmol/L), low-density lipoprotein cholesterol (LDL-C, $\mathrm{mmol} / \mathrm{L}$ ), and Lp-PLA2 (diluted 10-fold, ng/mL) were conducted. A chemiluminescent methodology was used to analyze the levels of ALT, AST, CREA, TG, TC, HDL-C, and LDL-C on the AU5800 autoanalyzer (Beckman Coulter, USA) via serum detection kits (Beckman Coulter, USA). Dry-type fluorescence immunity analysis (Guang Zhou Labsim Biotech Co., Ltd., 


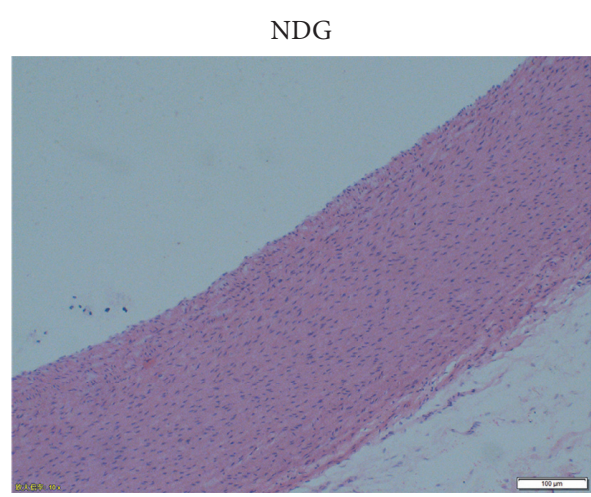

(a)

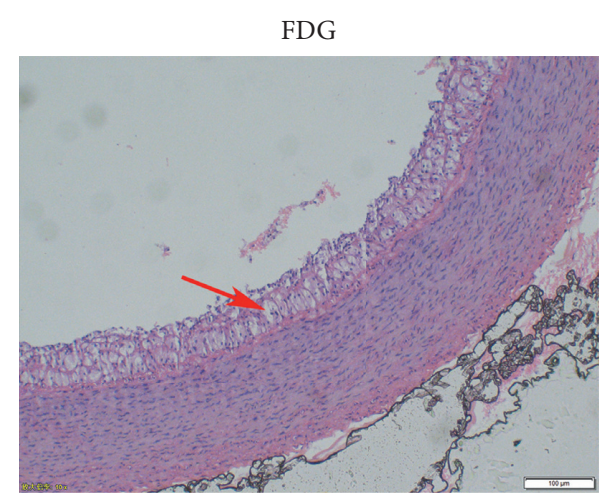

(b)

FigURE 1: Representative photomicrographs of aortic arch sections stained with hematoxylin-eosin of a normal diet group (NDG) and a fat diet group (FDG) in the preliminary experiment. Magnification: 10x. Bar represents $100 \mu \mathrm{m}$. The red arrow indicates that, compared with the NDG, foam cells formed under the intima in the FDG. Meanwhile, the intima was thickened, and plaques were formed. This showed that the atherosclerosis model was successfully established after a high-fat diet.

China) was performed to determinate the levels of Lp-PLA2 using a lipoprotein-related phospholipase A2 kit (Vazyme Biotech Co., Ltd., China), and the methodology was quantum dot fluorescence immunoassay.

2.4. Histological Analysis. After euthanasia of the rabbits by injection with sodium phenobarbital $(0.2 \mathrm{~g} / \mathrm{kg}$, diluted to a concentration of $3.5 \%$ ) into the ear vein, perpendicular to the vascular axis, the aortic arch was cut into approximately $2 \mathrm{~mm}$ lengths and placed in a test tube containing $4 \%$ paraformaldehyde. Samples were embedded in paraffin and stained with hematoxylin and eosin. Measurements of the thickness of the intima and smooth muscle layer were conducted by light microscopy (Olympus Corporation, Japan). The software connected with the microscope screenshot has a measurement tool. Clicking the measure button, the measured value will automatically appeared with dragging from a certain point to zero. After measuring the thickness of the intima and the smooth muscle layer under the microscope, the mean and standard deviations were determined, and the intima/middle layer ratio was subsequently calculated.

2.5. Statistical Analysis. All statistical analyses were performed using the SPSS 19.0 software (IBM, Armonk, New York); data from the experiments were analyzed and expressed as the mean \pm standard errors of the means (SEM). One-way analysis of variance (One way ANOVA) was used for further analysis. If the variance between groups was homogeneous, a comparison was conducted using the Bonferroni method, and the $P$ value was corrected. Dunnett's test method was adopted for cases of uneven variance.

\section{Results}

3.1. Laboratory Examinations. The comparison of weight, ALT, AST, CREA, TG, TC, HDL-C, LDL-C, and Lp-PLA2 levels between the three groups showed no significant difference at the baseline. The differences in pairwise comparison between the three groups also showed no significant difference at the baseline $(P<0.05)$ (Table 1$)$.

Intergroup comparison at the end of the 3-month experiment showed that the levels of AST, CREA, TC, HDL-C, LDL-C, and Lp-PLA2 were significantly different among the three groups $(P<0.05)$, as shown in Table 2 . In a pairwise comparison, CREA, TC, LDL-C, and Lp-PLA2 showed a significant difference between any two groups $(P<0.05)$. Additionally, AST $(77.91 \pm 24.54$ vs $40.51 \pm 6.46)$ and HDL-C $(1.58 \pm 0.35$ vs $1.03 \pm 0.20)$ were significantly different between RFG and NDG $(P<0.05)$. Meanwhile, HDL-C $(3.28 \pm 1.61$ vs $1.03 \pm 0.20)$ was also significantly different between the FDG and NDG groups $(P<0.05)$. No statistical significance in any other component was found with the pairwise comparison.

In addition, intragroup blood index comparisons were performed in the NDG, FDG, and RFG rabbits between the two time points: baseline and at 3 months. There were no significant differences in ALT, TG, LDL-C, and Lp-PLA2 levels between the baseline and after 3 months in NDG rabbits (Table 3). Meanwhile, the level of TG showed no significant difference in the FDG rabbits, and no significant difference in ALT in FDG and RFG was seen over the course of the experiment (Table 3 ).

3.2. Histologic Analysis. Figure 2 shows that the intima thickness of the aorta after RFG treatment was significantly decreased when compared with FDG, as was the ratio of the intima and aortic tunica media. The smooth muscle layers of the aortic arch in the three groups had no significant difference (Table 4).

\section{Discussion}

The prevention and treatment of cardiovascular and cerebrovascular diseases is an important topic of medical research, and studies have shown that AS participates in the development of many of these diseases [28]. In the present study, a stable rabbit AS model was established through 3 months of fat diet feeding. Utilizing this rabbit model, the 
TABLE 1: Biochemical analysis of rabbits from NDG $(n=8)$, FDG $(n=8)$, and RFG $(n=8)$ analyzed before the beginning of the fat diet (baseline).

\begin{tabular}{|c|c|c|c|c|c|}
\hline & NDG $(n=8)$ & FDG $(n=8)$ & RFG $(n=8)$ & $F$ & $P$ value \\
\hline Weight (kg) & $0.96 \pm 0.05$ & $0.99 \pm 0.08$ & $1.01 \pm 0.08$ & 0.903 & 0.420 \\
\hline ALT & $43.20 \pm 9.65$ & $41.22 \pm 8.83$ & $44.76 \pm 12.80$ & 0.226 & 0.800 \\
\hline AST & $36.38 \pm 8.61$ & $33.73 \pm 5.91$ & $34.88 \pm 4.98$ & 0.317 & 0.732 \\
\hline CREA & $67.94 \pm 8.39$ & $64.72 \pm 6.27$ & $65.75 \pm 9.69$ & 0.318 & 0.731 \\
\hline TG & $0.63 \pm 0.20$ & $0.50 \pm 0.16$ & $0.48 \pm 0.07$ & 2.230 & 0.132 \\
\hline $\mathrm{TC}$ & $1.94 \pm 0.21$ & $1.88 \pm 0.28$ & $1.99 \pm 0.43$ & 0.240 & 0.789 \\
\hline HDL-C & $0.93 \pm 0.19$ & $0.94 \pm 0.25$ & $0.93 \pm 0.17$ & 0.006 & 0.994 \\
\hline LDL-C & $0.84 \pm 0.22$ & $0.95 \pm 0.12$ & $0.80 \pm 0.27$ & 1.053 & 0.367 \\
\hline Lp-PLA2 $2^{\#}$ & $461.50 \pm 89.57$ & $525.48 \pm 32.50$ & $494.88 \pm 32.56$ & 2.424 & 0.113 \\
\hline
\end{tabular}

\#The test result of homogeneity of variance was $P<0.05$, and Dunnett's T method was used for multiple comparison of $P$ value correction, while the Bonferroni method was used for the other indicators. NDG, normal diet group; FDG, fat diet group; RFG, fat diet with resveratrol; ALT, alanine aminotransferase; AST, aspartate aminotransferase; CREA, creatinine; TG, triglycerides; TC, total cholesterol; HDL-C, high-density lipoprotein cholesterol; LDLC, low-density lipoprotein cholesterol; Lp-PLA2, lipoprotein-associated phospholipase A2.

TABLE 2: Biochemical analysis of rabbits from NDG $(n=8)$, FDG $(n=8)$, and RFG $(n=8)$ analyzed at the end of the 3-month fat diet.

\begin{tabular}{lcccc}
\hline & NDG $(n=8)$ & FDG $(n=8)$ & RFG $(n=8)$ & $F$ \\
\hline Weight $(\mathrm{kg})^{\#}$ & $2.90 \pm 0.08$ & $2.98 \pm 0.15$ & $2.96 \pm 0.07$ & 1.160 \\
ALT & $44.81 \pm 7.95$ & $50.86 \pm 24.53$ & $61.06 \pm 20.26$ & 1.506 \\
AST $^{\#}$ & $40.51 \pm 6.46$ & $84.32 \pm 54.94$ & $77.91 \pm 24.54^{\mathrm{a}}$ & 3.668 \\
CREA & $77.38 \pm 10.45$ & $111.92 \pm 11.38^{\mathrm{a}}$ & $127.63 \pm 10.84^{\mathrm{a}, \mathrm{b}}$ & 44.526 \\
TG & $0.62 \pm 0.21$ & $0.90 \pm 0.42$ & $0.76 \pm 0.28$ & 0.245 \\
TC $^{\#}$ & $2.19 \pm 0.30$ & $30.32 \pm 5.74^{\mathrm{a}}$ & $11.84 \pm 2.78^{\mathrm{a}, \mathrm{b}}$ & 1.567 \\
HDL $^{\#}$ & $1.03 \pm 0.20$ & $3.28 \pm 1.61^{\mathrm{a}}$ & $1.58 \pm 0.35^{\mathrm{a}}$ & 120.264 \\
LDL $_{\text {Lp-PLA2 }}$ & $0.91 \pm 0.22$ & $16.45 \pm 3.16^{\mathrm{a}}$ & $6.23 \pm 1.53^{\mathrm{a}, \mathrm{b}}$ & 11.946 \\
\hline
\end{tabular}

\#The test result of homogeneity of variance was $P<0.05$, and Dunnett's T method was used for multiple comparison of $P$ value correction, while the Bonferroni method was used for the other indicators. NDG, normal diet group; FDG, fat diet group; RFG, fat diet with resveratrol; ALT, alanine aminotransferase; AST, aspartate aminotransferase; CREA, creatinine; TG, triglycerides; TC, total cholesterol; HDL-C, high-density lipoprotein cholesterol; LDLC, low-density lipoprotein cholesterol; Lp-PLA2, lipoprotein-associated phospholipase A2. ${ }^{a}$ Statistically significant when compared to NDG at $P<0.05$. ${ }^{\mathrm{b}}$ Statistically significant when compared to FDG at $P<0.05$.

TABLE 3: Biochemical analysis of rabbits from RFG $(n=8)$ before the beginning of the treatment with fat diet and at the end of the 3-month fat diet.

\begin{tabular}{lccc}
\hline & Baseline $(n=8)$ & After 3 months $(n=8)$ & $t$ \\
\hline Weight(kg) (NDG) & $0.96 \pm 0.05$ & $2.90 \pm 0.08$ & -73.655 \\
Weight (kg) (FDG) & $0.99 \pm 0.08$ & $2.98 \pm 0.15$ & -49.925 \\
Weight (kg) (RFG) & $1.01 \pm 0.08$ & $2.96 \pm 0.07$ & -39.000 \\
ALT (NDG) & $43.20 \pm 9.65$ & $44.81 \pm 7.95$ & -0.781 \\
ALT (FDG) & $41.22 \pm 8.83$ & $50.86 \pm 24.53$ & -1.172 \\
ALT (RFG) & $44.76 \pm 12.80$ & $61.06 \pm 20.26$ & -2.160 \\
AST (NDG) & $36.38 \pm 8.61$ & $40.51 \pm 6.46$ & -2.490 \\
AST (FDG) & $33.73 \pm 5.91$ & $84.32 \pm 54.94$ & -2.639 \\
AST (RFG) & $34.88 \pm 4.98$ & $77.91 \pm 24.54$ & -5.587 \\
CREA (NDG) & $67.94 \pm 8.39$ & $77.38 \pm 10.45$ & -7.002 \\
CREA (FDG) & $64.72 \pm 6.27$ & $111.92 \pm 11.38$ & -10.794 \\
CREA (RFG) & $65.75 \pm 9.69$ & $127.63 \pm 10.84$ & -12.390 \\
TG (NDG) & $0.63 \pm 0.20$ & $0.62 \pm 0.21$ & 0.001 \\
TG (FDG) & $0.50 \pm 0.16$ & $0.90 \pm 0.42$ & 0.086 \\
TG (RFG) & $0.48 \pm 0.07$ & $0.76 \pm 0.28$ & -2.230 \\
TC (NDG) & $1.94 \pm 0.21$ & $2.19 \pm 0.30$ & -2.986 \\
TC (FDG) & $1.88 \pm 0.28$ & $30.32 \pm 5.74$ & $<0.001$ \\
TC (RFG) & $1.99 \pm 0.43$ & $11.84 \pm 2.78$ & $<0.001$ \\
HDL (NDG) & $0.93 \pm 0.19$ & $1.03 \pm 0.20$ & -2.919 \\
HDL (FDG) & $0.94 \pm 0.25$ & $3.28 \pm 1.61$ & -14.078 \\
HDL (RFG) & $0.93 \pm 0.17$ & $1.58 \pm 0.35$ & -9.275 \\
LDL (NDG) & $0.84 \pm 0.22$ & $0.91 \pm 0.22$ & -3.359 \\
LDL (FDG) & $0.95 \pm 0.12$ & $16.45 \pm 3.16$ & -3.815 \\
\hline
\end{tabular}


TABLE 3: Continued.

\begin{tabular}{|c|c|c|c|c|}
\hline & Baseline $(n=8)$ & After 3 months $(n=8)$ & $t$ & $P$ value \\
\hline LDL (RFG) & $0.80 \pm 0.27$ & $6.23 \pm 1.53$ & -9.221 & $<0.001$ \\
\hline Lp-PLA2 ${ }^{\#}(\mathrm{NDG})$ & $461.50 \pm 89.57$ & $520.14 \pm 51.55$ & -1.930 & 0.095 \\
\hline Lp-PLA2 ${ }^{\#}$ (FDG) & $525.48 \pm 32.50$ & $1928.88 \pm 385.78$ & -10.090 & $<0.001$ \\
\hline Lp-PLA2 ${ }^{\#}$ (RFG) & $494.88 \pm 32.56$ & $953.20 \pm 96.66$ & -12.816 & $<0.001$ \\
\hline
\end{tabular}

"The test result of homogeneity of variance was $P<0.05$, and Dunnett's T method was used for multiple comparison of $P$ value correction, while the Bonferroni method was used for the other indicators. NDG, normal diet group; FDG, fat diet group; RFG, fat diet with resveratrol; ALT, alanine aminotransferase; AST, aspartate aminotransferase; CREA, creatinine; TG, triglycerides; TC, total cholesterol; HDL-C, high-density lipoprotein cholesterol; LDLC, low-density lipoprotein cholesterol; Lp-PLA2, lipoprotein-associated phospholipase A2.

NDG

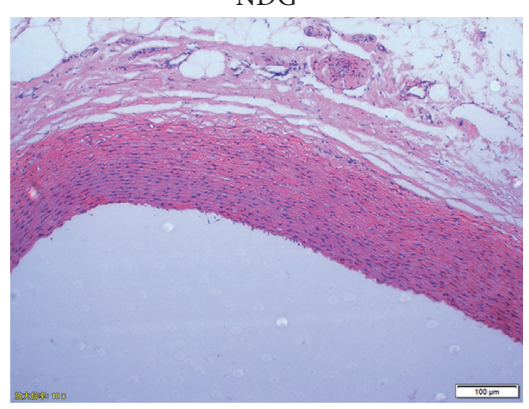

(a)
FDG

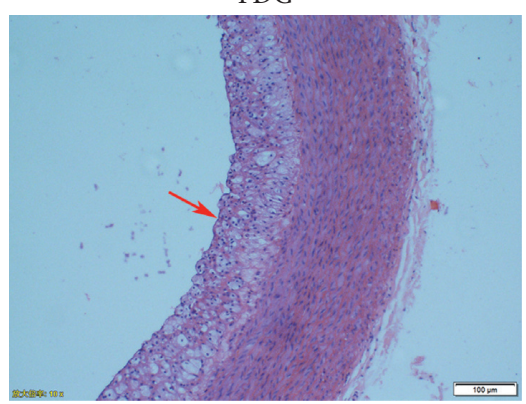

(b)
RFG

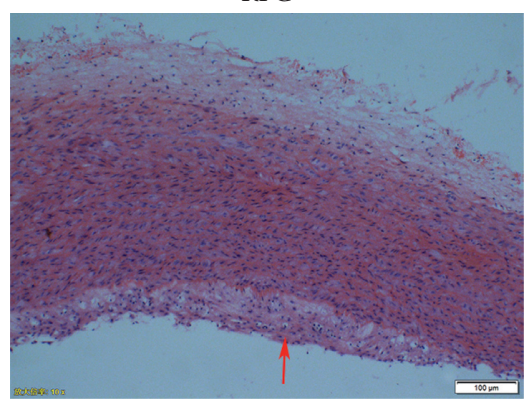

(c)

FIGURE 2: Representative photomicrographs of aortic arch sections stained with hematoxylin-eosin of a normal diet group (NDG), a fat diet group (FDG), and a resveratrol-treated FDG group (RFG). Magnification: 10x. Bar represents $100 \mu \mathrm{m}$. The red arrows indicate the thickening intima and plaque formation in the FDG and RFG, which were compared with the NDG. The intimal thickness of RGF was significantly lower than that of FDG $(P<0.05)$.

TABle 4: Pathological parameters of aortic arch atherosclerosis observed under the microscope of RFG and FDG.

\begin{tabular}{lcccc}
\hline & Group & Mean & SD $( \pm)$ & $P$ \\
\hline \multirow{2}{*}{ Intima } & FDG & 124.76 & 6.83 & 0.001 \\
& RFG & 52.44 & 14.94 & \\
\hline \multirow{2}{*}{ Smooth muscle layer } & FDG & 194.16 & 10.2 & 0.65 \\
& RFG & 173.48 & 4.05 & \\
\hline \multirow{2}{*}{ Intima/media ratio } & FDG & 0.64 & 0.04 & \multirow{2}{*}{0.001} \\
& RFG & 0.30 & 0.09 & \\
\hline
\end{tabular}

Unit, $\mu \mathrm{m}$; SD, standard deviation; FDG, fat diet group; RFG, fat diet with resveratrol.

relationship between resveratrol and AS was analyzed. Our results showed that the thickness of the intima and the ratio of the intima/media were significantly different in the RFG and FDG. Meanwhile, the value of CREA, TC, LDL-C, and Lp-PLA2 were significantly different between any two groups. Previous studies have shown that the formation of AS was accompanied by the proliferation of vascular endothelial cells and smooth muscle cells, among others [29]. By reducing the aortic intima area and the intima/media ratio, resveratrol inhibited the progression of atherosclerotic lesions [30]. In a study of the same rabbit model, resveratrol prevented the development of atherosclerotic lesions through the reduction of invasion of foam cells in the tunica media, [31]. In the present study, the thickness of the intima and the intima/media ratio were reduced by the intervention of resveratrol, which showed a significant difference between
RFG and FDG. The thickness of the smooth muscle layer was also reduced by resveratrol intervention, but the difference between RFG and FDG was not significant.

Some studies had found that Lp-PLA2 was associated with plaque progression and vulnerability $[32,33]$. In our study, Lp-PLA2 was significantly different in the RFG $(953.20 \pm 96.66)$ and FDG $(1928.88 \pm 385.78)$ groups when compared with the NDG $(520.14 \pm 51.55)$ group $(P<0.05)$. Meanwhile, with resveratrol intervention, LpPLA2 levels in the RFG were significantly lower than those in the FDG $(P<0.05)$. In circulating blood, LpPLA2 binds to lipoproteins through apolipoprotein (Apo) B, thereby hydrolyzing oxidized phospholipids in ox-LDL to produce lipid proinflammatory substances such as lysolecithin and oxidized free fatty acids [34]. These lipid proinflammatory substances can cause vascular endothelial cell death and endothelial dysfunction, stimulate the production of adhesion factors and cytokines, and produce atherosclerosis [16]. It is possible that resveratrol increases the expression of superoxide dismutase, catalase and glutathione peroxidase, and other antioxidant enzymes, thereby reducing the formation of free radicals, preventing endothelial damage, and directly blocking the inner membrane of blood vessels. In addition, resveratrol inhibits the production of proinflammatory cytokines and further reduces the atherosclerotic effect of Lp-PLA2 [35]. At the same time, resveratrol can inhibit the absorption of low-density lipoproteins by macrophages, prevent low-density 
lipoprotein peroxidation, inhibit lipid peroxidation, and regulate levels of blood lipids [36].

Lipid disorders are closely associated with the development of AS [37]. Studies have attributed the retention of serum lipoproteins in the artery wall to AS [38]. A cascade of chronic proinflammatory events in the artery wall were initiated by the accumulation of oxidized lipoproteins. The chronic inflammation observed in AS was caused by the recruitment of macrophages and the uptake of lipids into these cells [39]. According to previous studies, reduction of TC and LDL-C levels in patients with coronary heart disease could significantly reduce the mortality and recurrence rate of cardiovascular events $[40,41]$. In the present study, intervention with resveratrol significantly decreased the levels of TC and LDL-C in rabbits fed a high-fat diet. The mechanism by which resveratrol inhibits the formation of advanced atherosclerotic lesions appears to be through its inhibitory effects on LDL oxidation [42]. Resveratrol has been shown to protect lipids from peroxidative degradation and to stop the uptake of oxidized LDLs in the vascular wall in a concentration-dependent manner $[43,44]$. Thus, we hypothesized that resveratrol affected AS through impact on lipid TC and LDL-C levels.

AST and ALT enzymes are mainly concentrated in the liver and are involved in the conversion of sugars and proteins in vivo [45]. With the cell membrane fragmentation and permeability increase induced by liver cell damage, ALT and AST are activated and released into the blood. When liver cells are damaged, cells undergo degeneration, necrosis, and cell membrane fragmentation or permeability increase [46]. At this time, the ALT and AST contained in liver cells is released into the blood, thus increasing ALT and AST activity in the blood. Currently, AST and ALT are commonly used to detect liver injury in a clinic [37]. In the present study, a decrease in AST levels was detected in the comparison between RFG and FDG; however, this difference was not significant.

CREA is a product of human muscle metabolism, which is produced by the irreversible nonenzymatic dehydration of creatine [47]. The concentration of CREA in serum increased with the functional impairment of the kidney and is therefore a reliable indicator of renal function [48]. Studies have shown that lipid metabolism disorder is a factor in the development of chronic kidney disease, and resultant kidney damage can aggravate the dysregulation of lipid metabolism [49]. CREA has been widely used as a common test for kidney injury $[50,51]$. In our study, CREA values increased with resveratrol treatment in rabbits fed a high-fat diet $(P<0.05)$. Resveratrol has previously been reported to cause nephrotoxicity [52]. The possible reason behind this is that the kidneys are the dominant excretion organ, and the recovery rate of total resveratrol in the urine and feces fluctuates between $70 \%$ and $98 \%$ within 24 hours. Therefore, in our study, compared with the FDG, the CREA values were significantly increased in RFG $(P<0.05)$.

In summary, the present study suggests that resveratrol could inhibit the incrassation of arterial intima. In addition, resveratrol treatment reduced the levels of TC, LDLC, Lp-PLA2, and CREA in rabbits fed a high-fat diet. Thus, resveratrol may have a function in preventing AS. However, clinical trials are needed to further investigate this effect.

\section{Conclusions}

Resveratrol might have antiatherosclerosis effect on a rabbit model of AS.

\section{Data Availability}

The data used to support the findings of this study are included within the article.

\section{Additional Points}

Highlights. (1) Resveratrol reduced the thickness of the aortic intima and the ratio of intima/media. (2) Lp-PLA2 levels were significantly reduced by resveratrol. (3) TC, LDL-C, and CREA levels were significantly different in RFG and FDG.

\section{Conflicts of Interest}

The authors declare that there are no conflicts of interest regarding the publication of this paper.

\section{Acknowledgments}

This wok was supported by the Jilin Province Natural Science Foundation (no. 20160101073JC).

\section{References}

[1] D. Xi, J. Zhao, W. Lai, and Z. Guo, "Systematic analysis of the molecular mechanism underlying atherosclerosis using a text mining approach," Human Genomics, vol. 10, no. 1, p. 14, 2016.

[2] G. K. Hansson, "Inflammation, atherosclerosis, and coronary artery disease," New England Journal of Medicine, vol. 352, no. 16, pp. 1685-1695, 2005.

[3] M. Awad, P. Eshtehardi, and L. J. Shaw, "Imaging atherosclerosis for global predictive health and wellness," JACC: Cardiovascular Imaging, vol. 9, no. 5, pp. 577-579, 2016.

[4] K. T. Howitz, K. J. Bitterman, H. Y. Cohen et al., "Small molecule activators of sirtuins extend Saccharomyces cerevisiae lifespan," Nature, vol. 425, no. 6954, pp. 191-196, 2003.

[5] T. Wilson, T. J. Knight, D. C. Beitz, D. S. Lewis, and R. L. Engen, "Resveratrol promotes atherosclerosis in hypercholesterolemic rabbits," Life Sciences, vol. 59, no. 1, pp. PL15-PL21, 1996.

[6] S. S. Kang, M. Cuendet, D. C. Endringer, V. L. Croy, J. M. Pezzuto, and M. A. Lipton, "Synthesis and biological evaluation of a library of resveratrol analogues as inhibitors of COX-1, COX-2 and NF- $\kappa \mathrm{B}, "$ Bioorganic \& Medicinal Chemistry, vol. 17, no. 3, pp. 1044-1054, 2009.

[7] G. Spanier, H. Xu, N. Xia, S. Tobias, and H. Li, "Resveratrol reduces endothelial oxidative stress by modulating the gene expression of superoxide dismutase 1 (SOD1), glutathione peroxidase 1 (GPx1) and NADPH oxidase subunit (NOX4)," Journal of Physiology and Pharmacology: An Official Journal of 
the Polish Physiological Society, vol. 60, no. 4, pp. 111-116, 2009.

[8] S. Timmers, E. Konings, L. Bilet et al., "Calorie restriction-like effects of 30 days of resveratrol supplementation on energy metabolism and metabolic profile in obese humans," Cell Metabolism, vol. 14, no. 5, pp. 612-622, 2011.

[9] H. Zhang, J. Zhang, Z. Ungvari, and C. Zhang, "Resveratrol improves endothelial function: role of TNF $\alpha$ and vascular oxidative stress," Arteriosclerosis, Thrombosis, and Vascular Biology, vol. 29, no. 8, pp. 1164-1171, 2009.

[10] M. Y. Shen, G. Hsiao, C. L. Liu et al., "Inhibitory mechanisms of resveratrol in platelet activation: pivotal roles of p38 MAPK and NO/cyclic GMP," British Journal of Haematology, vol. 139, no. 3, 2007.

[11] H. Berrougui, G. Grenier, S. Loued, G. Drouin, and A. Khalil, "A new insight into resveratrol as an atheroprotective compound: inhibition of lipid peroxidation and enhancement of cholesterol efflux," Atherosclerosis, vol. 207, no. 2, pp. 420427, 2009.

[12] D.-W. Park, K. Baek, J.-R. Kim et al., "Resveratrol inhibits foam cell formation via NADPH oxidase 1-mediated reactive oxygen species and monocyte chemotactic protein-1," Experimental and Molecular Medicine, vol. 41, no. 3, p. 171, 2009.

[13] M. S. Sabatine, D. A. Morrow, and M. O’Donoghue, "Prognostic utility of lipoprotein-associated phospholipase A2 for cardiovascular outcomes in patients with stable coronary artery disease," Arteriosclerosis, Thrombosis, and Vascular Biology, vol. 27, no. 11, pp. 2463-2469, 2007.

[14] S. Tsimikas, J. Willeit, M. Knoflach et al., "Lipoprotein-associated phospholipase A2 activity, ferritin levels, metabolic syndrome, and 10-year cardiovascular and non-cardiovascular mortality: results from the Bruneck study," European Heart Journal, vol. 30, no. 1, pp. 107-115, 2008.

[15] A. Rubinstein and E. Izkhakov, "Lipoprotein associated phospholipase A2," Harefuah, vol. 150, no. 2, pp. 136-140, 2011.

[16] K. Sudhir, "Lipoprotein-associated phospholipase A2, a novel inflammatory biomarker and independent risk predictor for cardiovascular disease," The Journal of Clinical Endocrinology \& Metabolism, vol. 90, no. 5, pp. 3100-3105, 2005.

[17] S. Sun, M. Zhang, Q. Yang et al., "Resveratrol suppresses lipoprotein-associated phospholipase A2 expression by reducing oxidative stress in macrophages and animal models," Molecular Nutrition \& Food Research, vol. 61, no. 10, Article ID 1601112, 2017.

[18] Y. Luo, Q. Fei, W. M. Botello-Smith et al., "Resveratrol protects membranes from PLA1 and PLA2 hydrolytic attack," Biophysical Journal, vol. 114, no. 3, p. 259a, 2018.

[19] Y. Wang, S. Hu, L. Ren et al., "Lp-PLA2 as a risk factor of early neurological deterioration in acute ischemic stroke with TOAST type of large arterial atherosclerosis," Neurological Research, vol. 41, no. 1, pp. 1-8, 2019.

[20] Y. Tian, H. Jia, S. Li et al., "The associations of stroke, transient ischemic attack, and/or stroke-related recurrent vascular events with lipoprotein-associated phospholipase A2," Medicine, vol. 96, no. 51, Article ID e9413, 2017.

[21] M. Murakami and I. Kudo, "Phospholipase A2," Journal of Biochemistry, vol. 131, no. 3, p. 285, 2002.

[22] M. J. Caslake and C. J. Packard, "Lipoprotein-associated phospholipase A2 as a biomarker for coronary disease and stroke," Nature Clinical Practice Cardiovascular Medicine, vol. 2, no. 10, pp. 529-535, 2005.

[23] Y. G. Sun, J. Xu, M. D. Jensen, and A. Simonyi, "Phospholipase A2 in the central nervous system: implications for neurodegenerative diseases," Journal of Lipid Research, vol. 45, no. 2, pp. 205-213, 2004.

[24] N. S. V. Ditzhuijzen, H. Mvd, O. Sorop et al., "Invasive coronary imaging in animal models of atherosclerosis," Netherlands Heart Journal, vol. 19, no. 10, pp. 442-446, 2011.

[25] B. C. Meneghini, E. R. Tavares, M. C. Guido et al., "Lipid core nanoparticles as vehicle for docetaxel reduces atherosclerotic lesion, inflammation, cell death and proliferation in an atherosclerosis rabbit model," Vascular Pharmacology, vol. 115, pp. 46-54, 2019.

[26] L. Zarei, M. Bahrami, N. Farhad, S. M. A. Froushani, and A. Abbasi, "All-trans retinoic acid effectively reduces atheroma plaque size in a rabbit model of high-fat-induced atherosclerosis," Advances in Clinical and Experimental Medicine, vol. 27, no. 12, pp. 1631-1636, 2018.

[27] J.-G. Zou, Z.-R. Wang, Y.-Z. Huang, K.-J. Cao, and J. M. Wu, "Effect of red wine and wine polyphenol resveratrol on endothelial function in hypercholesterolemic rabbits," International Journal of Molecular Medicine, vol. 11, no. 3, pp. 317-320, 2003.

[28] K. K. Lau, Y. K. Wong, Y. H. Chan et al., "Visit-to-visit blood pressure variability as a prognostic marker in patients with cardiovascular and cerebrovascular diseases--relationships and comparisons with vascular markers of atherosclerosis," Atherosclerosis, vol. 235, no. 1, pp. 230-235, 2014.

[29] C. Mcnamara, I. Sarembock, B. Bachhuber et al., "Thrombin and vascular smooth muscle cell proliferation: implications for atherosclerosis and restenosis," Seminars in Thrombosis \& Hemostasis, vol. 22, no. 2, pp. 139-144, 1996.

[30] R. S. Matos, L. A. V. Baroncini, L. B. Précoma et al., "Resveratrol causes antiatherogenic effects in an animal model of atherosclerosis," Arquivos Brasileiros de Cardiologia, vol. 98, no. 2, pp. 136-142, 2012.

[31] M. Castro, M. M. R. Pacheco, and M. R. F. Machado, "Morphology of aortic arch in rabbits with atherosclerosis treated with resveratrol," International Journal of Applied Research in Veterinary Medicine, vol. 7, no. 4, pp. 190-195, 2009.

[32] A. A. Farooqui and L. A. Horrocks, "Plasmalogens, phospholipase A2, and docosahexaenoic acid turnover in brain tissue," Journal of Molecular Neuroscience, vol. 16, no. 2-3, pp. 263-272, 2001.

[33] B. S. Cummings, J. Mchowat, and R. G. Schnellmann, "Role of an endoplasmic reticulum $\mathrm{Ca}^{2+}$-independent phospholipase A2 in cisplatin-induced renal cell apoptosis," Journal of Pharmacology and Experimental Therapeutics, vol. 308, no. 3, pp. 921-928, 2004.

[34] M. Kim, M. Kim, Y. J. Lee et al., "Effects of $\alpha$-linolenic acid supplementation in perilla oil on collagen-epinephrine closure time, activated partial thromboplastin time and Lp-PLA2 activity in non-diabetic and hypercholesterolaemic subjects," Journal of Functional Foods, vol. 23, pp. 95-104, 2016.

[35] K. Rosing, M. Fobker, F. Kannenberg et al., "Everolimus therapy is associated with reduced lipoprotein-associated phospholipase A2 (Lp-Pla2) activity and oxidative stress in heart transplant recipients," Atherosclerosis, vol. 230, no. 1, pp. 164-170, 2013.

[36] L. Frémont, L. Belguendouz, and S. Delpal, "Antioxidant activity of resveratrol and alcohol-free wine polyphenols related to LDL oxidation and polyunsaturated fatty acids," Life Sciences, vol. 64, no. 26, pp. 2511-2521, 1999.

[37] W. R. Kim, S. L. Flamm, A. M. D. Bisceglie, and H. C. Bodenheimer, "Serum activity of alanine 
aminotransferase (ALT) as an indicator of health and disease," Hepatology, vol. 47, no. 4, pp. 1363-1370, 2008.

[38] J. K. Moore and M. W. Freeman, "Scavenger receptors in atherosclerosis: beyond lipid uptake," Arteriosclerosis, Thrombosis, and Vascular Biology, vol. 26, no. 8, pp. 17021711, 2006.

[39] G. Obermayer, T. Afonyushkin, and C. J. Binder, "Oxidized low density lipoprotein in inflammation-driven thrombosis," Journal of Thrombosis \& Haemostasis, vol. 16, no. 3, pp. 418-428, 2018.

[40] N. Nakaya, K. Mizuno, Y. Ohashi, T. Teramoto, and H. Nakamura, "Low-dose pravastatin and age-related differences in risk factors for cardiovascular disease in hypercholesterolaemic Japanese," Drugs \& Aging, vol. 28, no. 9, pp. 681-692, 2011.

[41] J. Shepherd, S. M. Cobbe, I. Ford et al., "Prevention of coronary heart disease with pravastatin in men with hypercholesterolemia," Atherosclerosis Supplements, vol. 5, no. 3, pp. 91-97, 2004.

[42] E. Fan, L. Zhang, S. Jiang, and Y. Bai, "Beneficial effects of resveratrol on atherosclerosis," Journal of Medicinal Food, vol. 11, no. 4, pp. 610-614, 2008.

[43] S. V. Nigdikar, N. R. Williams, B. A. Griffin, and A. N. Howard, "Consumption of red wine polyphenols reduces the susceptibility of low-density lipoproteins to oxidation in vivo," The American Journal of Clinical Nutrition, vol. 68 , no. 2, pp. 258-265, 1998.

[44] D. Shanmuganayagam, T. F. Warner, C. G. Krueger, J. D. Reed, and J. D. Folts, "Concord grape juice attenuates platelet aggregation, serum cholesterol and development of atheroma in hypercholesterolemic rabbits," Atherosclerosis, vol. 190, no. 1, pp. 135-142, 2007.

[45] N. Cheng, N. Ren, H. Gao, X. Lei, J. Zheng, and W. Cao, "Antioxidant and hepatoprotective effects of Schisandra chinensis pollen extract on CCl4-induced acute liver damage in mice," Food \& Chemical Toxicology, vol. 55, pp. 234-240, 2013.

[46] A. Amin and A. A. Hamza, "Oxidative stress mediates druginduced hepatotoxicity in rats: a possible role of DNA fragmentation," Toxicology, vol. 208, no. 3, pp. 367-375, 2005.

[47] C. J. Kochansky and T. G. Strein, "Determination of uremic toxins in biofluids: creatinine, creatine, uric acid and xanthines," Journal of Chromatography B: Biomedical Sciences and Applications, vol. 747, no. 1-2, pp. 217-227, 2000.

[48] C. Couchoud, N. Pozet, M. Labeeuw, and C. Pouteil-Noble, "Screening early renal failure: cut-off values for serum creatinine as an indicator of renal impairment," Kidney International, vol. 55, no. 5, pp. 1878-1884, 1999.

[49] J. C. Rutledge, K. F. Ng, H. H. Aung, and D. W. Wilson, "Role of triglyceride-rich lipoproteins in diabetic nephropathy," Nature Reviews Nephrology, vol. 6, no. 6, pp. 361-370, 2010.

[50] S. S. Waikar and J. V. Bonventre, "Creatinine kinetics and the definition of acute kidney injury," Journal of the American Society of Nephrology, vol. 20, no. 3, pp. 672-679, 2009.

[51] S. S. Waikar, R. A. Betensky, and J. V. Bonventre, Creatinine as the Gold Standard for Kidney Injury Biomarker Studies?, Oxford University Press, Oxford, UK, 2009.

[52] J. A. Crowell, P. J. Korytko, R. L. Morrissey, T. D. Booth, and B. S. Levine, "Resveratrol-associated renal toxicity," Toxicological Sciences, vol. 82, no. 2, pp. 614-619, 2004. 